\title{
Urban Monitoring of Unpleasant Odors with a Handheld Electronic Nose
}

\author{
Andres Gongora \\ MAPIR Group \\ University of Malaga - IBIMA \\ Malaga, Spain \\ andresgongora@uma.es
}

\author{
Alberto Jaenal \\ MAPIR Group \\ UMA - IBIMA \\ Malaga, Spain \\ David Chaves \\ MAPIR Group \\ UMA - IBIMA \\ Malaga, Spain \\ albertojaenal@uma.es davidchaves@uma.es jgmonroy@uma.es \\ Javier Monroy \\ MAPIR Group \\ UMA - IBIMA \\ Malaga, Spain
}

\author{
Javier Gonzalez-Jimenez \\ MAPIR Group \\ UMA - IBIMA \\ Malaga, Spain \\ javiergonzalez@uma.es
}

\begin{abstract}
This work describes a real application of artificial olfaction where a handheld electronic nose was used as a validation tool for a chemical spillage in a southern town in Spain. The objective was to check if the palliative and precautionary measurements taken by the authorities were working effectively, removing the elevated values of phenol that were detected in a wide area of the municipality of Coria del Rio (Spain). To this end, a gas distribution map of the affected neighborhoods was built with a portable electronic nose taking into consideration the likely presence of other volatile chemicals in the area. For the latter, we trained a volatile chemical classifier with a dataset of typical urban smells that we wanted to remove from the results (e.g. traffic emissions, garbage, fresh-air), as well as with a specific air-born phenol dataset. Results demonstrated that the palliative measures were in general satisfactory, but some hot-spots were located where the intensity of phenol-like smell was still higher than desired. Advice was given to the local authorities to doublecheck these locations with analytical gas-monitoring equipment.

Index Terms-electronic nose, gas classification, smell map
\end{abstract}

\section{INTRODUCTION}

In the late summer of 2017, the neighbors of Coria del Río (South Spain) noticed an unpleasant odor in the ambient air. They described it as a gasoline or plastic-like smell, yet none they could easily trace or identify [1]. The Regional Ministry of the Environment started consequently an investigation to determine its cause and reach, and revealed shortly after that the odor was caused by a mix of various chemical substances in the town's sewer network. In a few, specific spots, it was measured abnormal levels of phenol and diphenylamine [2] as well as other fuel related compounds in lesser concentration [3]. However, they were unable to trace the exact location at which the substances were leaking into the sewers, or determine whether someone was pouring them intentionally.

In the meantime, the town's council proceeded to take measures to mitigate the bad odors: seals on all rain gutters and a powerful extractor-fan to vent the sewers in a controlled manner. The so generated negative air-pressure was meant to prevent further smells from entering the affected homes through their drainage system. The air extractor was placed next to the town's river, so that the strong winds that flow over it could drag the expelled gases away.

Funded by the Governments of Spain and Andalusia, and the European Regional Development Fund under projects TEP2012-530 and FPU17/04512.

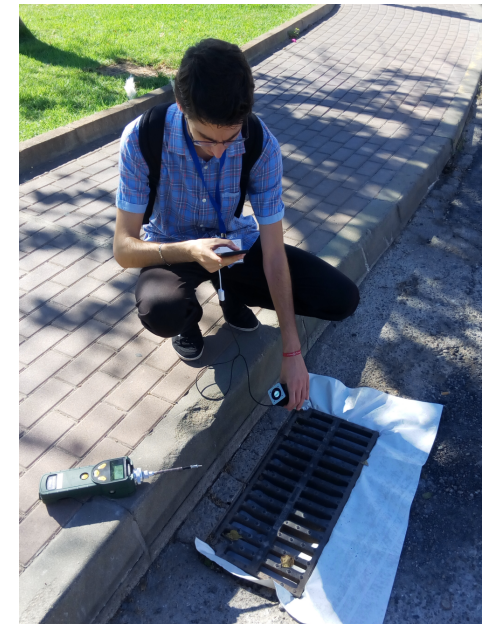

(a) Sample data acquisition

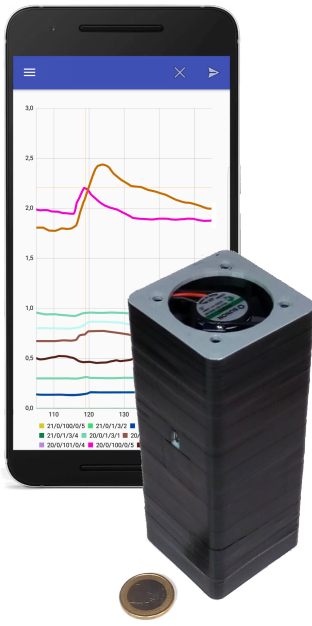

(b) E-nose and application
Figure 1: Field-trip to Coria del Río to record a georeferenced dataset with the e-nose and smartphone application shown in (b). As can be observed in (a), the local Council had covered all manholes and rain gutters to prevent the phenol-like smells from escaping the sewers.

Given peculiarity of the situation, our research group was contacted to help in the evaluation of the efficiency of the aforementioned adopted measures and, if that was the case, to report on any noteworthy locations that might require additional analysis. We inspected to that end the affected zones with a portable electronic nose (e-nose) and logged its raw output into a georeferenced dataset. Thereafter, we classified all the gathered data with Deep Learning to detect phenol-like smells and represented them in an odor distribution map [4]. This map served to assess if any perceptible odor remained, and whether the air extractor diffused the phenol leak over unpopulated areas, as intended.

\section{Methodology}

The goal of this work is to estimate a map of fuel-like smells in the ambient air of the town Coria del Río to provide a general overview of their distribution. Ideally, this could have been accomplished by taking individual air-samples 
around town to reveal their exact composition (e.g. through chromatography-mass spectrometry [5]). Nevertheless, this kind of procedure is intractable for wide areas if high spatial resolution is required [6]. Hereof, e-noses are a better option because they allow for continuous measurements that, paired with an appropriate classifier, can infer the smell of the sampled air [4]. And even though e-noses might be unable to estimate the exact gas-concentration under this conditions [7], their output is still sufficient for our purposes.

Accordingly, we proceeded to record the output of a portable e-nose during an approximately 6 hours long walk (from noon until dusk on the 26th October 2017), during which we repeatedly passed the same locations to ensure consistent readings. All readings were logged on a custom smartphone application that connected to the e-nose, georeferencing the sensor readings by means of the phone's GPS coordinates.

Also, because the smell was attributed to phenol, a gas heavier than air, we carried the e-nose below waist height at all times and brought it close to the floor at the locations of interests (e.g. sewers, around the extractor, etc.) as shown in Fig. 1a.

We then trained a Convolutional Neural Network (CNN) with two dataset that we had previously recorded for the same e-nose. The first contained over 450 samples of typical urban smells [4] (e.g. fresh air, traffic emissions, garbage, etc.), whereas the second was specifically created using phenol at different concentrations and ambient conditions (temperature, humidity, and pressure). Finally, we classified each visited location according to the presence of phenol-like smells, and drew them on a map as intended.

We describe next each of these tools in more detail.

\section{A. Electronic Nose and Smartphone Application}

The employed e-nose is based on a modular design meant for lightweight and portable setups [8]. It carries three metaloxide (MOX) sensors by Figaro (TGS2600, TGS2602, and TGS2611), 3 MOX sensors by SGX (dual MICS-4514, and MICS-5524), one amperometric electrochemical (AEC) sensor by Alphasense (SO2-B4), as well as one relative-humidity and two temperature sensors. Please note that none of these are specifically meant to measure phenol-like substances. However, they all cover a wide range of organic compounds, meaning that their combined response is suited to detect these kind of volatiles as shown experimentally.

As depicted in Fig. 1b, we carried the e-nose within a tubular enclosure with a fan, thus improving the response time of its sensors. Also, by connecting it over USB-OTG to our custom smartphone-application [4], we were able to visualize all data in real-time; but most importantly, it took care of combining the e-nose's output with the phone's GPS, and logging it (at a $1 \mathrm{~Hz}$ rate) for later analysis.

\section{B. Smell Classifier}

We considered several machine learning algorithms for the required smell classification, including decision trees, linear discriminant analysis, and support vector machines. Yet as

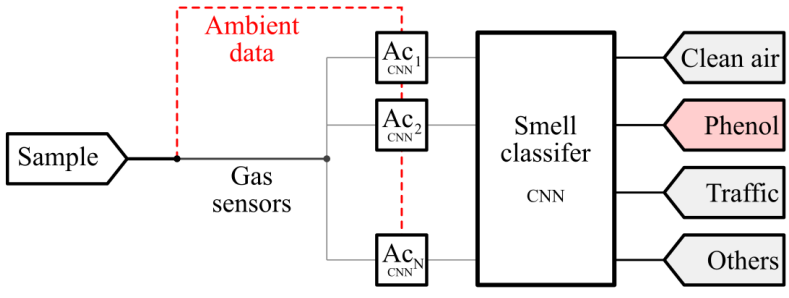

Figure 2: The employed $\mathrm{CCN}$ architecture minimizes the correlation effect between sensor data and ambient conditions. It relies on the preconditioning $\mathrm{CNNs} A c_{i}$ to compensate temperature and humidity drift before the classification.

shown in our previous work [4], a time-window based CNN offers the bests results for our particular configuration.

Notwithstanding, we have refined our previous classifier for this work with a preconditioning architecture as shown in Fig. 2. Each of the submodules $A c_{i}$ is a CNN in its own right, and is meant to compensate humidity and temperature drift of its associated gas-sensor. This modification favours a better generalization of the training labels (fresh air, phenol, urban smells, and others) from the ambient conditions; which provides more robust results than our previous approach.

\section{Smell-Map Generation}

The smell-map is meant to show an estimation of where the smell of phenol was most intense. To that end We take our classifier's prediction for phenol-like smells and scale it by the e-nose's absolute output signal (measured with respect to its baseline). Thus, the map shows greater values for those samples that measured a high gas concentrations, and at the same time, got a low classification uncertainty. Finally, we plot each sample with a weighted Gaussian function to account for GPS error and to combine overlapping measurements from multiple passes [9].

\section{RESULTS}

After gathering and classifying the smells in the streets of Coria del Río, we obtained the phenol smell map shown in Fig. 3. Please note that it does not represent the true concentration of ambient phenol, but an estimate of its intensity as sensed by our e-nose and classifier. Nonetheless, it remained an useful tool to assess the situation and to advise the other teams of where to emplace their more precise equipment.

As shown on the map, the highest phenol intensity lied right next to the extractor on the river promenade. Given the lack of nearby buildings to block wind currents, the gas spread southward and away from the population as intended. On the other hand, we hardly detected any phenol-like smells within the residential area other than at three places. The first one was in front of some of the buildings whose inhabitants were most distressed by the situation; and who kept their windows open because their houses' draining pipes had no isolation siphons. Whereas the other two were rain gutters (one next to the aforementioned homes) that someone had uncovered. 


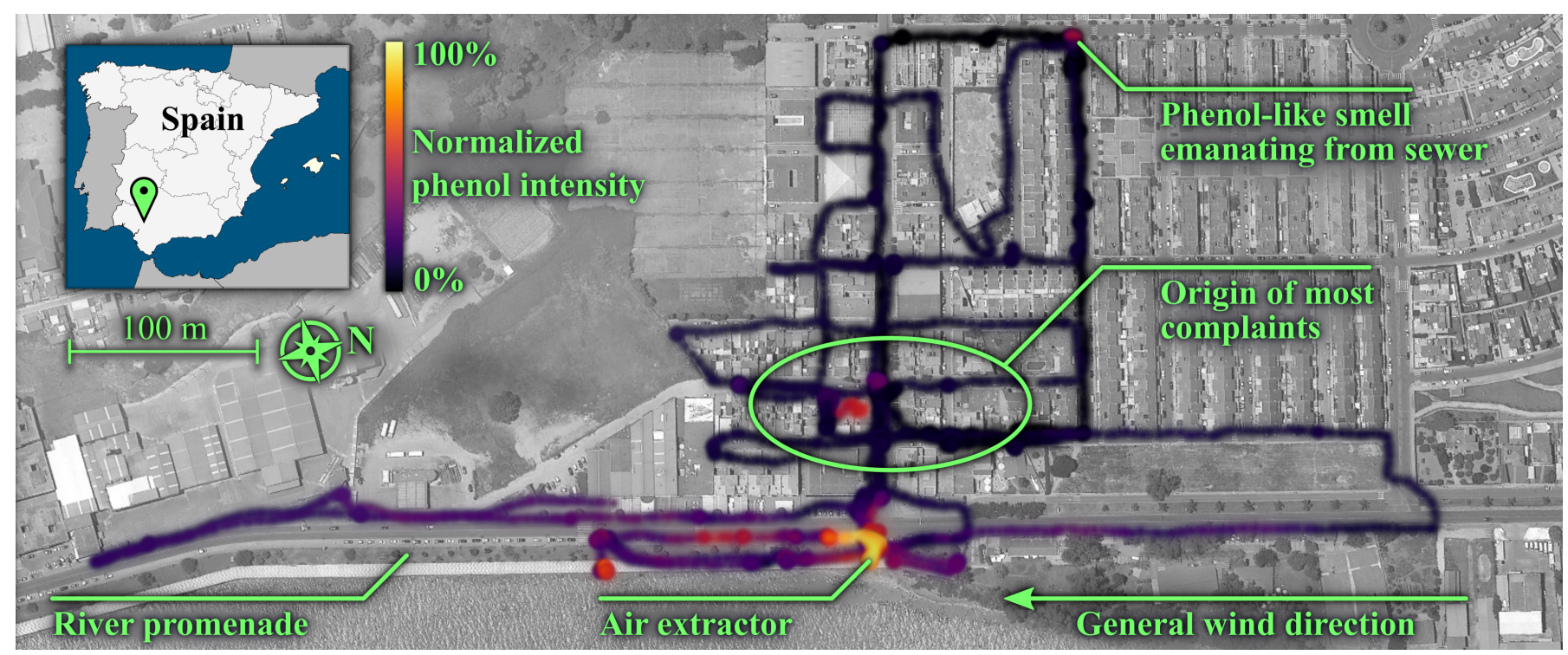

Figure 3: Normalized phenol-like smell estimation map of Coria del Río. The displayed estimation corresponds to the classifier's output, scaled by the absolute measured intensity with respect to an initial base line for clean air. All sampled locations are displayed in a scale that ranges from black (no phenol detected) to maximum gas estimation with a 100\% certainty; notice however that the later is never achieved. The highest value lies around $94 \%$ next to the extractor, and at 30-45\% at for a few specific locations of the residential area. The wind blew mostly southward, but not with much force.

It is therefore reasonable to say that, for the most part, the council's palliative measures were successful. Naturally, they were only meant to alleviate the situation while the actual cause of the problem was solved.

\section{CONCLUSIONS AND Future WORK}

This work shows that e-noses are a convenient tool to assess the spatial distribution of smells with a high resolution and in a short time. Particularly, for our application we were able to determine that the majority of Coria del Río had been cleansed from the phenol-like smells, and that the extractor was expelling them to the river basin as intended.

Nonetheless, this technology is still limited in that it is unable to recover the exact concentration of the gases of interest unless a thorough calibration is done and the appropriate reading corrections are adopted to account for environmental conditions and sensor drift. Accordingly, our next steps will focus on developing a method to calibrate the e-nose and classifier to respond only to a very specific compound; or alternatively, on introducing the information of exact gas measurements into the smell-mapping process to attain an exact prediction.

As for the origin of the gases, it still remains uncertain. Shortly after our field trip, the rain removed the smells and have hindered all subsequent analysis ever since (including the summer of 2018). Notwithstanding, as the cause of the leakage, the latest data and investigation pointed to a former industrial cesspit located below the neighbourhood [10].

\section{REFERENCES}

[1] "Unease because of a strong fuel-like smell in a neighbourhood of coria del río [news article in Spanish]," https://sevilla.abc.es/provincia/ aljarafe/sevi-inquietud-fuerte-olor-gasoil-barriada-guadalquivir-coria201710172344_noticia.html, (Accessed: 2018-12-11).

[2] "The Regional Ministry of the Environment and Territorial Organization of Andalusia will analyze the air quality in coria del río after an episode of strong odors [news article in Spanish]," http://www.juntadeandalucia.es/presidencia/portavoz/medioambiente/ 126648/medio/ambiente/realizara/analisis/calidad/aire/zona/coria/ detectado/episodio/fuertes/olores, (Accessed: 2018-12-11).

[3] "The sewer contamination in a town of Sevilla causes the neighbours health problems [news article in Spanish]," https://www.eldiario.es/andalucia/alcantarillado-Coria-contaminadasustancias-industrial_0_700280546.html, (Accessed: 2018-12-11).

[4] A. Gongora, D. Chaves, A. Jaenal, J. Monroy, and J. Gonzalez-Jimenez, "Toward the generation of smell maps: Matching electro-chemical sensor information with human odor perception," in Frontiers in Artificial Intelligence and Applications, vol. 310. IOS Press, 2018.

[5] S. Cui, J. Wang, L. Yang, J. Wu, and X. Wang, "Qualitative and quantitative analysis on aroma characteristics of ginseng at different ages using e-nose and gc-ms combined with chemometrics," Journal of pharmaceutical and biomedical analysis, vol. 102, pp. 64-77, 2015.

[6] E. O. Gaga, T. Döğeroğlu, Ö. Özden, A. Ari, O. D. Yay, H. Altuğ, N. Akyol, S. Örnektekin, and W. Van Doorn, "Evaluation of air quality by passive and active sampling in an urban city in turkey: current status and spatial analysis of air pollution exposure," Environmental Science and Pollution Research, vol. 19, no. 8, pp. 3579-3596, 2012.

[7] F. Röck, N. Barsan, and U. Weimar, "Electronic nose: current status and future trends," Chemical reviews, vol. 108, no. 2, pp. 705-725, 2008.

[8] A. Gongora, J. Monroy, and J. Gonzalez-Jimenez, "An electronic architecture for multi-purpose artificial noses," Journal of Sensors, feb 2018.

[9] J. G. Monroy, J.-L. Blanco, and J. Gonzalez-Jimenez, "Time-variant gas distribution mapping with obstacle information," Autonomous Robots, vol. 40, no. 1, pp. 1-16, 2016.

[10] "Latests investigations are very close to finding the origin of coria's gases [news article in Spanish]," https://sevilla.abc.es/provincia/aljarafe/ sevi-investigaciones-cerca-hallar-origen-gases-coria-201807311759_ noticia.html, (Accessed: 2018-12-11). 